GLASNIK MATEMATIČKI

Vol. 40(60)(2005), $87-102$

\title{
APPROXIMATION IN SMIRNOV-ORLICZ CLASSES
}

\author{
Daniyal M. Israfilov, Burcin Oktay and Ramazan Akgun \\ Balikesir University, Turkey
}

\begin{abstract}
We use the approximation properties of the Faber polynomials to obtain some direct theorems of the polynomial approximation in Smirnov-Orlicz classes.
\end{abstract}

\section{Introduction and Main Results}

Let $\Gamma$ be a rectifiable Jordan curve in the complex plane $\mathbb{C}$ and $G:=$ Int $\Gamma, G^{-}:=\operatorname{Ext} \Gamma$. Without loss of generality we may assume $0 \in G$. Let $\mathbb{T}:=\{w \in \mathbb{C}:|w|=1\}, \mathbb{D}:=\operatorname{Int} \mathbb{T}$ and $\mathbb{D}^{-}:=\operatorname{Ext} \mathbb{T}$. Let also $w=\varphi(z)$ be the conformal mapping of $G^{-}$onto $\mathbb{D}^{-}$, normalized by

$$
\varphi(\infty)=\infty, \lim _{z \rightarrow \infty} \frac{\varphi(z)}{z}>0,
$$

and let $\psi$ be its inverse.

If $1 \leq p<\infty$, we denote by $L_{p}(\Gamma)$ and $E_{p}(G)$ the set of all measurable complex valued functions $f$ on $\Gamma$ such that $|f|^{p}$ is Lebesgue integrable with respect to arclength, and the Smirnov class of analytic functions in $G$, respectively. Since $\Gamma$ is rectifiable, we have that $\varphi^{\prime} \in E_{1}\left(G^{-}\right)$and $\psi^{\prime} \in E_{1}\left(\mathbb{D}^{-}\right)$, and hence the functions $\varphi^{\prime}$ and $\psi^{\prime}$ admit non-tangential limits almost everywhere (a.e.) on $\Gamma$ and on $\mathbb{T}$, and these functions belong to $L_{1}(\Gamma)$ and $L_{1}(\mathbb{T})$, respectively (see, for example $[10, p .419]$ ).

Let $h$ be a continuous function on $[0,2 \pi]$. Its modulus of continuity is defined by

$$
\omega(t, h):=\sup \left\{\left|h\left(t_{1}\right)-h\left(t_{2}\right)\right|: t_{1}, t_{2} \in[0,2 \pi],\left|t_{1}-t_{2}\right| \leq t\right\}, \quad t \geq 0 .
$$

2000 Mathematics Subject Classification. 30E10, 41A10, 41A20, 41A25, 46E30.

Key words and phrases. Dini-smooth curve, Smirnov-Orlicz classes, polynomial approximation, Faber polynomials, maximal convergence. 
The function $h$ is called Dini-continuous if

$$
\int_{0}^{\pi} t^{-1} \omega(t, h) d t<\infty
$$

Definition 1.1 ([17, p. 48]). The curve $\Gamma$ is called Dini-smooth if it has a parametrization

$$
\Gamma: \varphi_{0}(\tau), \quad 0 \leq \tau \leq 2 \pi
$$

such that $\varphi_{0}^{\prime}(\tau)$ is Dini-continuous and $\neq 0$.

If $\Gamma$ is Dini-smooth, then [21]

$$
0<c_{1} \leq\left|\varphi^{\prime}(z)\right| \leq c_{2}<\infty, \quad z \in \Gamma
$$

for some constants $c_{1}$ and $c_{2}$ independent of $z$.

A function $M(u): \mathbb{R} \rightarrow \mathbb{R}^{+}$, where $\mathbb{R}:=(-\infty, \infty)$ and $\mathbb{R}^{+}:=(0, \infty)$, is called an $N-$ function if it admits of the representation

$$
M(u)=\int_{0}^{|u|} p(t) d t
$$

where the function $p(t)$ is right continuous and nondecreasing for $t \geq 0$ and positive for $t>0$, which satisfies the conditions

$$
p(0)=0, p(\infty):=\lim _{t \rightarrow \infty} p(t)=\infty .
$$

The function

$$
N(v):=\int_{0}^{|v|} q(s) d s
$$

where

$$
q(s):=\sup _{p(t) \leq s} t, \quad(s \geq 0)
$$

is defined as complementary function of $M(u)[16$, p. 11].

Let $M$ be an $N$ - function and $N$ be its complementary function. By $L_{M}(\Gamma)$ we denote the linear space of Lebesgue measurable functions $f: \Gamma \rightarrow \mathbb{C}$ satisfying the condition

$$
\int_{\Gamma} M[\alpha|f(z)|]|d z|<\infty
$$

for some $\alpha>0$.

The space $L_{M}(\Gamma)$ becomes a Banach space with the norm

$$
\|f\|_{L_{M}(\Gamma)}:=\sup \left\{\int_{\Gamma}|f(z) g(z)||d z|: g \in L_{N}(\Gamma), \rho(g ; N) \leq 1\right\},
$$


where

$$
\rho(g ; N):=\int_{\Gamma} N[|g(z)|]|d z| .
$$

The norm $\|\cdot\|_{L_{M}(\Gamma)}$ is called Orlicz norm and the Banach space $L_{M}(\Gamma)$ is called Orlicz space. Every function in $L_{M}(\Gamma)$ is integrable on $\Gamma[18$, p. 50], i.e.

$$
L_{M}(\Gamma) \subset L_{1}(\Gamma)
$$

An $N$ - function $M$ satisfies the $\Delta_{2}-$ condition if

$$
\limsup _{x \rightarrow \infty} \frac{M(2 x)}{M(x)}<\infty .
$$

The Orlicz space $L_{M}(\Gamma)$ is reflexive if and only if the $N$ - function $M$ and its complementary function $N$ both satisfy the $\Delta_{2}-$ condition [18, p. 113].

Let $\Gamma_{r}$ be the image of the circle $\{w \in \mathbb{C}:|w|=r, 0<r<1\}$ under some conformal mapping of $\mathbb{D}$ onto $G$ and let $M$ be an $N$-function.

DEFINITION 1.2. If an analytic function $f$ in $G$ satisfies

$$
\int_{\Gamma_{r}} M[|f(z)|]|d z|<\infty
$$

uniformly in $r$, it belongs to Smirnov-Orlicz class $E_{M}(G)$.

If $M(x)=M(x, p):=x^{p}, 1<p<\infty$, then the Smirnov-Orlicz class $E_{M}(G)$ coincides with the usual Smirnov class $E_{p}(G)$.

Every function in the class $E_{M}(G)$ has [14] the non-tangential boundary values a.e. on $\Gamma$ and the boundary function belongs to $L_{M}(\Gamma)$, and hence for $f \in E_{M}(G)$ we can define the $E_{M}(G)$ norm as:

$$
\|f\|_{E_{M}(G)}:=\|f\|_{L_{M}(\Gamma)} .
$$

For $\varsigma \in \Gamma$ we define the point $\varsigma_{h} \in \Gamma$ by

$$
\varsigma_{h}:=\psi\left(\varphi(\varsigma) e^{i h}\right), \quad h \in[0,2 \pi],
$$

and also the shift $T_{h} f$ for $f \in L_{M}(\Gamma)$ as:

$$
T_{h} f(\varsigma):=f\left(\varsigma_{h}\right), \quad \varsigma \in \Gamma \text {. }
$$

Using relation (1), it can be easily verified that, if $\Gamma$ is Dini-smooth, then $L_{M}(\Gamma)$ is invariant under the shift $T_{h} f$.

We define the modulus of continuity for $f \in L_{M}(\Gamma)$ as:

$$
\omega_{M}(\delta, f):=\sup _{|h| \leq \delta}\left\|f-T_{h} f\right\|_{L_{M}(\Gamma)}, \quad \delta \geq 0
$$

which satisfies the conditions

$$
\begin{gathered}
\omega_{M}(0, f)=0 \\
\omega_{M}(\delta, f) \geq 0 \text { for } \delta>0,
\end{gathered}
$$




$$
\begin{gathered}
\lim _{\delta \rightarrow 0} \omega_{M}(\delta, f)=0, \\
\omega_{M}(\delta, f+g) \leq \omega_{M}(\delta, f)+\omega_{M}(\delta, g)
\end{gathered}
$$

for $f, g \in E_{M}(G)$.

For $f \in E_{M}(G)$ we put

$$
\begin{aligned}
E_{n}^{M}(f, G):=\inf \left\|f-p_{n}\right\|_{L_{M}(\Gamma)} \\
(5) \quad \inf \left\{\sup \left\{\int_{\Gamma}\left|\left(f(\varsigma)-p_{n}(\varsigma)\right) g(\varsigma)\right||d \varsigma| ; \rho(g ; N) \leq 1\right\}\right\},
\end{aligned}
$$

where inf is taken over the polynomials $p_{n}$ of degree at most $n$.

In this work, we considered some problems of the polynomial approximation in Smirnov-Orlicz class $E_{M}(G)$. Our new results are the following.

TheOrem 1.3. Let $G$ be a finite simply connected domain with the Dinismooth boundary $\Gamma$, and let $E_{M}(G)$ be a reflexive Smirnov-Orlicz space on $G$. Then for every $f \in E_{M}(G)$ and any natural number $n$ there exists an algebraic polynomial $p_{n}(\cdot, f)$ of degree at most $n$ such that

$$
\left\|f-p_{n}(\cdot, f)\right\|_{L_{M}(\Gamma)} \leq c \omega_{M}\left(\frac{1}{n}, f\right)
$$

with some constant $c$ independent of $n$.

In the more general case, namely when $\Gamma$ is a Carleson curve, applying the same method of summation, but using different modulus of continuity some direct theorem of approximation theory by polynomials in SmirnovOrlicz class $E_{M}(G)$ is given in [8]. The modulus of continuity $\omega_{M}$, used in this work, is simplier than the modulus of continuity considered in [8].

Similar problems for the spaces $L_{p}(\Gamma)$ and $E_{p}(G), 1 \leq p<\infty$, have been studied in $[1,2,4,5,10,11,12,15]$. All these results were proved under different restrictive conditions on $\Gamma=\partial G$.

Some inverse problems of approximation theory in Smirnov-Orlicz classes have been investigated by Kokilashvili [14] in the case that $\Gamma$ is Dini-smooth.

Now let $K$ be a bounded continuum with the connected complement $D:=\overline{\mathbb{C}} \backslash K$ and let $f(z)$ be an analytic function on $K$. It is well known that $[20$, p. 199] the expansion

$$
f(z)=\sum_{k=0}^{\infty} a_{k} \Phi_{k}(z), \quad z \in K
$$

with the Faber coefficients

$$
a_{k}:=\frac{1}{2 \pi i} \int_{\mathbb{T}} \frac{f(\psi(t))}{t^{k+1}} d t, \quad k=0,1,2, \ldots,
$$


converges absolutely and uniformly on $K$, where $\Phi_{k}(z), k=0,1,2, \ldots$, are the Faber polynomials for $K$ that satisfy the relation

$$
\frac{\psi^{\prime}(w)}{\psi(w)-z}=\sum_{k=0}^{\infty} \frac{\Phi_{k}(z)}{w^{k+1}}, \quad z \in K,|w|>1 .
$$

The detailed information about the Faber polynomials and their approximation properties can be found in the monographs $[8,19,20]$.

Let us introduce the value

$$
R_{n}(z, f):=f(z)-\sum_{k=0}^{n} a_{k} \Phi_{k}(z)=\sum_{k=n+1}^{\infty} a_{k} \Phi_{k}(z), \quad z \in K,
$$

and put

$$
\Gamma_{R}:=\{z \in D:|\varphi(z)|=R\} \text { and } G_{R}:=\operatorname{Int} \Gamma_{R}, R>1 .
$$

The following theorem characterizes the maximal convergence property of the Faber series (6) in the Smirnov-Orlicz space $E_{M}\left(G_{R}\right)$.

Theorem 1.4. If $f \in E_{M}\left(G_{R}\right), R>1$, then

$$
\left|R_{n}(z, f)\right| \leq \frac{c}{R^{n+1}(R-1)} E_{n}^{M}\left(f, G_{R}\right) \sqrt{n \ln n}, \quad z \in K
$$

with a constant $c>0$ independent of $n$ and $z$.

From theorem 1.3 and 1.4, we have the following corollary.

COROLlary 1.5. Let $K$ be a continuum with connected complement and let $E_{M}\left(G_{R}\right)$ be a reflexive Smirnov-Orlicz class on $G_{R}, R>1$. If $f \in E_{M}\left(G_{R}\right)$, then

$$
\left|R_{n}(z, f)\right| \leq \frac{c}{R^{n+1}(R-1)} \omega_{M}\left(\frac{1}{n}, f\right) \sqrt{n \ln n}, \quad z \in K,
$$

with $c>0$.

Theorem 1.4 in the Smirnov spaces $E_{p}(G), p>1$, was proved in [20, p. 207].

We use $c, c_{1}, c_{2}, \ldots$ to denote constants ( which may, in general, differ in different relations) depending only on numbers that are not important for the question of interest.

\section{Auxiliary Results}

Let $\Gamma$ be a rectifiable Jordan curve and $f \in L_{1}(\Gamma)$. The functions $f^{+}$and $f^{-}$defined by

$$
f^{+}(z)=\frac{1}{2 \pi i} \int_{\Gamma} \frac{f(\varsigma)}{\varsigma-z} d \varsigma, \quad z \in G
$$


and

$$
f^{-}(z)=\frac{1}{2 \pi i} \int_{\Gamma} \frac{f(\varsigma)}{\varsigma-z} d \varsigma, \quad z \in G^{-},
$$

are analytic in $G$ and $G^{-}$, respectively and $f^{-}(\infty)=0$.

Let also

$$
S_{\Gamma} f\left(z_{0}\right):=\lim _{\varepsilon \rightarrow 0} \int_{\Gamma \cap\left\{\varsigma:\left|\varsigma-z_{0}\right| \geq \varepsilon\right\}} \frac{f(\varsigma)}{\varsigma-z_{0}} d \varsigma, \quad z_{0} \in \Gamma
$$

be the Cauchy singular integral of $f \in L_{1}(\Gamma)$.

If one of the functions $f^{+}$or $f^{-}$has non-tangential limits a.e. on $\Gamma$, then $S_{\Gamma} f(z)$ exists a.e. on $\Gamma$ and also the other one has non-tangential limits a.e. on $\Gamma$. Conversely, if $S_{\Gamma} f(z)$ exist a.e. on $\Gamma$, then both functions $f^{+}$and $f^{-}$have non-tangential limits a.e. on $\Gamma$. In both cases, the formulae

$$
\begin{aligned}
& f^{+}(z)=S_{\Gamma} f(z)+\frac{1}{2} f(z) \\
& f^{-}(z)=S_{\Gamma} f(z)-\frac{1}{2} f(z)
\end{aligned}
$$

hold, and hence

$$
f=f^{+}-f^{-}
$$

a.e. on $\Gamma[9$, p. 431].

The linear operator $S_{\Gamma}: f \rightarrow S_{\Gamma} f$ is called the Cauchy singular operator.

For $z \in \Gamma$ and $\epsilon>0$, let $\Gamma(z, \epsilon)$ denote the portion of $\Gamma$ which is inside the open disk of radius $\epsilon$ centered at $z$, i.e. $\Gamma(z, \epsilon):=\{t \in \Gamma:|t-z|<\epsilon\}$. Further, let $|\Gamma(z, \epsilon)|$ denote the length of $\Gamma(z, \epsilon)$. A rectifiable Jordan curve $\Gamma$ is called a Carleson curve if

$$
\sup _{\epsilon>0} \sup _{z \in \Gamma} \frac{1}{\epsilon}|\Gamma(z, \epsilon)|<\infty .
$$

THEOREM 2.1 ([13]). Let $\Gamma$ be a rectifiable Jordan curve and let $L_{M}(\Gamma)$ be a reflexive Orlicz space on $\Gamma$. Then the singular operator $S_{\Gamma}$ is bounded on $L_{M}(\Gamma)$, i.e.

$$
\left\|S_{\Gamma} f\right\|_{L_{M}(\Gamma)} \leq c_{3}\|f\|_{L_{M}(\Gamma)} \text { for all } f \in L_{M}(\Gamma)
$$

for some constant $c_{3}>0$, if and only if $\Gamma$ is a Carleson curve.

TheOREm $2.2([16$, p. 67]). For every pair of real valued functions $u(z) \in$ $L_{M}(\Gamma), v(z) \in L_{N}(\Gamma)$ the inequality

$$
\int_{\Gamma} u(z) v(z) d z \leq \rho(u ; M)+\rho(v ; N)
$$

holds. 
Theorem 2.3 ([16, p. 74]). For every pair of real valued functions $u(z) \in$ $L_{M}(\Gamma), v(z) \in L_{N}(\Gamma)$ the inequality

$$
\left|\int_{\Gamma} u(z) v(z) d z\right| \leq\|u\|_{L_{M}(\Gamma)}\|v\|_{L_{N}(\Gamma)}
$$

holds.

The coefficients of the series in (6) are determined by the formulae

$$
a_{k}:=\frac{1}{2 \pi i} \int_{\Gamma} \frac{f(\varsigma) \varphi^{\prime}(\varsigma)}{\varphi^{k+1}(\varsigma)} d \varsigma=\frac{1}{2 \pi i} \int_{\mathbb{T}} \frac{f(\psi(t))}{t^{k+1}} d t, \quad k=0,1,2, \ldots,
$$

and hence the relation (8) implies that

$$
R_{n}(z, f)=\frac{1}{2 \pi i} \int_{\mathbb{T}} f(\psi(t))\left[\sum_{k=n+1}^{\infty} \frac{\Phi_{k}(z)}{t^{k+1}}\right] d t .
$$

If $p_{n}(z)$ is a polynomial of degree at most $n$, then

$$
R_{n}(z, f)=\frac{1}{2 \pi i} \int_{\mathbb{T}}\left\{f(\psi(t))-p_{n}(\psi(t))\right\}\left[\sum_{k=n+1}^{\infty} \frac{\Phi_{k}(z)}{t^{k+1}}\right] d t .
$$

Since

$$
\Phi_{k}(z)=[\varphi(z)]^{k}+E_{k}(z), \quad z \in K,
$$

where $E_{k}(z)$ is analytic on the whole domain $D$ and $E_{k}(\infty)=0$, we have

$$
\sum_{k=n+1}^{\infty} \frac{\Phi_{k}(z)}{t^{k+1}}=\sum_{k=n+1}^{\infty} \frac{[\varphi(z)]^{k}}{t^{k+1}}+\sum_{k=n+1}^{\infty} \frac{E_{k}(z)}{t^{k+1}}
$$

Hence from (15), taking into account (16), we get

$$
\begin{aligned}
& \left|R_{n}(z, f)\right| \leq \frac{1}{2 \pi} \int_{|t|=R}\left|f(\psi(t))-p_{n}(\psi(t))\right|\left|\sum_{k=n+1}^{\infty} \frac{w^{k}}{t^{k+1}}\right||d t| \\
& +\frac{1}{2 \pi} \int_{|t|=R}\left|f(\psi(t))-p_{n}(\psi(t))\right|\left|\sum_{k=n+1}^{\infty} E_{k}(\psi(w)) \frac{1}{t^{k+1}}\right||d t| .
\end{aligned}
$$

We shall also use the relations

$$
E_{k}(\psi(w))=\frac{1}{2 \pi i} \int_{\Gamma} \tau^{k} F(\tau, w) d \tau, \quad|w| \geq r>1,
$$


and

$$
\frac{1}{2 \pi i} \int_{\Gamma}|F(\tau, w)||d \tau| \leq \sqrt{\frac{r^{2}}{r^{4}-1} \ln \frac{r^{2}}{r^{2}-1}}, \quad r>1,|w| \geq r>1,
$$

given in [20, p. 63-205], where

$$
F(\tau, w):=\frac{\psi^{\prime}(\tau)}{\psi(\tau)-\psi(w)}-\frac{1}{\tau-w}, \quad|\tau|>1, \quad|w|>1 .
$$

\section{Proofs of Main Results}

Proof of Theorem 1.3. Let $f \in L_{M}(\Gamma)$. Then by (2) we have $f \in$ $L_{1}(\Gamma)$. Since $\Gamma$ is Dini-smooth, we have $f \circ \psi \in L_{1}(\mathbb{T})$ and hence we can associate a formal series

$$
\sum_{k=0}^{\infty} a_{k} w^{k}+\sum_{k=1}^{\infty} \frac{b_{k}}{w^{k}}
$$

with the function $f \circ \psi \in L_{1}(\mathbb{T})$, i.e.

$$
f(\psi(w)) \backsim \sum_{k=0}^{\infty} a_{k} w^{k}+\sum_{k=1}^{\infty} \frac{b_{k}}{w^{k}} .
$$

Let

$$
K_{n}(\theta)=\sum_{m=-n}^{n} \lambda_{m}^{(n)} e^{i m \theta}
$$

be an even, nonnegative trigonometric polynomial satisfying the conditions

$$
\begin{gathered}
\frac{1}{2 \pi} \int_{-\pi}^{\pi} K_{n}(\theta) d \theta=1, \\
\int_{0}^{\pi} \theta K_{n}(\theta) d \theta \leq \frac{c_{4}}{n}
\end{gathered}
$$

for every natural number $n$ and with some constant $c_{4}>0$ (for example, the Jackson kernel

$$
J_{n}(\theta):=\frac{3\left(\sin \frac{n \theta}{2}\right)^{4}}{n\left(2 n^{2}+1\right)\left(\sin \frac{\theta}{2}\right)^{4}}
$$

satisfies the above cited conditions, see [6, p. 203-204]).

Consider the integral

$$
I(\theta, z):=\frac{1}{2 \pi i} \int_{\Gamma} \frac{f\left(\varsigma_{-}\right)}{\varsigma-z} d \varsigma, \quad z \in G .
$$


Using the change of variables $\varsigma=\psi\left(e^{i t}\right)$, we obtain

$$
I(\theta, z):=\frac{1}{2 \pi i} \int_{-\pi}^{\pi} f\left(\psi\left(e^{i(t-\theta)}\right)\right) \frac{\psi^{\prime}\left(e^{i t}\right) e^{i t}}{\psi\left(e^{i t}\right)-z} d t
$$

and taking into account the relations (20) and (7), we can write

$$
I(\theta, z) \backsim \sum_{k=0}^{\infty} a_{k} \Phi_{k}(z) e^{-i k \theta} .
$$

Since $I(\theta, z) \in L_{1}([-\pi, \pi])$ and $K_{n}(\theta)$ is of bounded variation, by the generalized Parseval identity [3, p. 225-228], we obtain

$$
\frac{1}{2 \pi} \int_{-\pi}^{\pi} K_{n}(\theta) I(\theta, z) d \theta=\sum_{k=0}^{n} \lambda_{k}^{(n)} a_{k} \Phi_{k}(z)
$$

which together with (23) implies that

$$
\frac{1}{4 \pi^{2} i} \int_{-\pi}^{\pi} K_{n}(\theta) d \theta \int_{\Gamma} \frac{f\left(\varsigma_{-\theta}\right)}{\varsigma-z} d \varsigma=\sum_{k=0}^{n} \lambda_{k}^{(n)} a_{k} \Phi_{k}(z), \quad z \in G .
$$

Hence we see that

$$
P_{n}(z, f):=\frac{1}{4 \pi^{2} i} \int_{-\pi}^{\pi} K_{n}(\theta) d \theta \int_{\Gamma} \frac{f\left(\varsigma_{-\theta}\right)}{\varsigma-z} d \varsigma, \quad z \in G,
$$

is an algebraic polynomial of degree $n$.

Since the kernel $K_{n}(\theta)$ is an even function, we have

$$
P_{n}(z, f)=\frac{1}{4 \pi^{2} i} \int_{0}^{\pi} K_{n}(\theta) d \theta \int_{\Gamma}\left[f\left(\varsigma_{\theta}\right)+f\left(\varsigma_{(-\theta)}\right)\right] \frac{d \varsigma}{\varsigma-z},
$$

and by (3) and (9), we conclude that

$$
\begin{aligned}
P_{n}(z, f) & =\frac{1}{4 \pi^{2} i} \int_{0}^{\pi} K_{n}(\theta) d \theta \int_{\Gamma}\left[T_{\theta} f(\varsigma)+T_{(-\theta)} f(\varsigma)\right] \frac{d \varsigma}{\varsigma-z} \\
& =\frac{1}{2 \pi} \int_{0}^{\pi} K_{n}(\theta)\left[\left(T_{\theta} f\right)^{+}(z)+\left(T_{(-\theta)} f\right)^{+}(z)\right] d \theta, \quad z \in G .
\end{aligned}
$$

Now let $f \in E_{M}(G)$ and $z^{\prime} \in G$. Multiplying both side of the equality

$$
\frac{1}{2 \pi} \int_{-\pi}^{\pi} K_{n}(\theta) d \theta=1
$$


by $f^{+}\left(z^{\prime}\right)$ we get

$$
f\left(z^{\prime}\right)=f^{+}\left(z^{\prime}\right)=\frac{1}{2 \pi} \int_{-\pi}^{\pi} f^{+}\left(z^{\prime}\right) K_{n}(\theta) d \theta=\frac{1}{2 \pi} \int_{0}^{\pi} 2 f^{+}\left(z^{\prime}\right) K_{n}(\theta) d \theta
$$

and hence

$$
\begin{aligned}
f\left(z^{\prime}\right) & -P_{n}\left(z^{\prime}, f\right) \\
= & \frac{1}{2 \pi} \int_{0}^{\pi} K_{n}(\theta)\left\{2 f^{+}\left(z^{\prime}\right)-\left[\left(T_{\theta} f\right)^{+}\left(z^{\prime}\right)+\left(T_{(-\theta)} f\right)^{+}\left(z^{\prime}\right)\right]\right\} d \theta .
\end{aligned}
$$

Taking the limit $z^{\prime} \rightarrow z \in \Gamma$ along all nontangential paths inside $\Gamma$ and using (10), we obtain

$$
\begin{aligned}
f(z)-P_{n}(z, f)= & \frac{1}{2 \pi} \int_{0}^{\pi} K_{n}(\theta)\left[2 S_{\Gamma} f(z)+f(z)-S_{\Gamma}\left(T_{\theta} f\right)(z)-\frac{1}{2} T_{\theta} f(z)\right. \\
& \left.-S_{\Gamma}\left(T_{(-\theta)} f\right)(z)-\frac{1}{2}\left(T_{(-\theta)} f\right)(z)\right] d \theta \\
= & \frac{1}{2 \pi} \int_{0}^{\pi} K_{n}(\theta)\left[S_{\Gamma}\left(f-T_{\theta} f\right)(z)+S_{\Gamma}\left(f-T_{(-\theta)} f\right)(z)\right] d \theta \\
& +\frac{1}{4 \pi} \int_{0}^{\pi} K_{n}(\theta)\left[\left(f-T_{\theta} f\right)(z)+\left(f-T_{(-\theta)} f\right)(z)\right] d \theta
\end{aligned}
$$

for almost all $z \in \Gamma$.

Taking the supremum over all functions $g \in L_{N}(\Gamma)$ with $\rho(g ; N) \leq 1$ in the last relation, we have

$$
\begin{aligned}
& \left\|f-P_{n}(\cdot, f)\right\|_{L_{M}(\Gamma)}=\sup \int_{\Gamma}\left|f(z)-P_{n}(z, f)\right||g(z)||d z| \\
& \leq \sup \int_{\Gamma}\left|\frac{1}{2 \pi} \int_{0}^{\pi} K_{n}(\theta)\left[S_{\Gamma}\left(f-T_{\theta} f\right)(z)+S_{\Gamma}\left(f-T_{(-\theta)} f\right)(z)\right] d \theta\right||g(z)||d z| \\
& \quad+\sup _{\Gamma}\left|\frac{1}{4 \pi} \int_{0}^{\pi} K_{n}(\theta)\left[\left(f-T_{\theta} f\right)(z)+\left(f-T_{(-\theta)} f\right)(z)\right] d \theta\right||g(z)||d z|
\end{aligned}
$$




$$
\begin{aligned}
& \leq \sup \int_{\Gamma}\left\{\frac { 1 } { 2 \pi } \int _ { 0 } ^ { \pi } K _ { n } ( \theta ) \left(\left|S_{\Gamma}\left(f-T_{\theta} f\right)(z)\right|\right.\right. \\
& \left.\left.+\left|S_{\Gamma}\left(f-T_{(-\theta)} f\right)(z)\right|\right) d \theta\right\}|g(z)||d z| \\
& +\sup \int_{\Gamma}\left\{\frac { 1 } { 4 \pi } \int _ { 0 } ^ { \pi } K _ { n } ( \theta ) \left[\left|\left(f-T_{\theta} f\right)(z)\right|\right.\right. \\
& \left.\left.+\left|\left(f-T_{(-\theta)} f\right)(z)\right|\right] d \theta\right\}|g(z)||d z|,
\end{aligned}
$$

and by Fubini's theorem

$$
\begin{aligned}
& \left\|f-P_{n}(\cdot, f)\right\|_{L_{M}(\Gamma)} \\
& \leq \frac{1}{2 \pi} \int_{0}^{\pi} K_{n}(\theta)\left\{\operatorname { s u p } \int _ { \Gamma } \left[\left|S_{\Gamma}\left(f-T_{\theta} f\right)(z)\right|\right.\right. \\
& \left.\left.\quad+\left|S_{\Gamma}\left(f-T_{(-\theta)} f\right)(z)\right|\right]|g(z)||d z|\right\} d \theta \\
& \quad+\frac{1}{4 \pi} \int_{0}^{\pi} K_{n}(\theta)\left\{\operatorname { s u p } \int _ { \Gamma } \left[\left|\left(f-T_{\theta} f\right)(z)\right|\right.\right. \\
& \left.\left.\quad+\left|\left(f-T_{(-\theta)} f\right)(z)\right|\right]|g(z)||d z|\right\} d \theta \\
& \leq \frac{1}{2 \pi} \int_{0}^{\pi} K_{n}(\theta)\left[\left\|S_{\Gamma}\left(f-T_{\theta} f\right)\right\|_{L_{M}(\Gamma)}+\left\|S_{\Gamma}\left(f-T_{(-\theta)} f\right)\right\|_{L_{M}(\Gamma)}\right] d \theta \\
& \quad+\frac{1}{4 \pi} \int_{0}^{\pi} K_{n}(\theta)\left[\left\|f-T_{\theta} f\right\|_{L_{M}(\Gamma)}+\left\|f-T_{(-\theta)} f\right\|_{L_{M}(\Gamma)}\right] d \theta .
\end{aligned}
$$

Now applying (11), we get

$$
\left\|f-P_{n}(\cdot, f)\right\|_{L_{M}(\Gamma)} \leq c_{5} \int_{0}^{\pi} K_{n}(\theta)\left[\left\|f-T_{\theta} f\right\|_{L_{M}(\Gamma)}+\left\|f-T_{(-\theta)} f\right\|_{L_{M}(\Gamma)}\right] d \theta,
$$

and recalling the definition (4) of $\omega_{M}(\delta, f)$, we obtain

$$
\begin{aligned}
\left\|f-P_{n}(\cdot, f)\right\|_{L_{M}(\Gamma)} & \leq c_{6} \int_{0}^{\pi} K_{n}(\theta) \omega_{M}(\theta, f) d \theta \\
& \leq c_{7} \omega_{M}\left(\frac{1}{n}, f\right) \int_{0}^{\pi} K_{n}(\theta)(n \theta+1) d \theta .
\end{aligned}
$$


Consequently from (21) and (22), we have

$$
\left\|f-P_{n}(\cdot, f)\right\|_{L_{M}(\Gamma)} \leq c_{8} \omega_{M}\left(\frac{1}{n}, f\right),
$$

which proves Theorem 1.3.

Proof of Theorem 1.4. Let $z \in \Gamma_{r}, 1<r<R$ and $p_{n}(z)$ be the best approximating polynomial of degree at most $n$ to the function $f \in E_{M}\left(G_{R}\right)$. Denoting

$$
\begin{aligned}
& I_{1}:=\frac{1}{2 \pi} \int_{|t|=R}\left|f(\psi(t))-p_{n}(\psi(t))\right|\left|\sum_{k=n+1}^{\infty} \frac{w^{k}}{t^{k+1}}\right||d t|, \\
& I_{2}:=\frac{1}{2 \pi} \int_{|t|=R}\left|f(\psi(t))-p_{n}(\psi(t))\right|\left|\sum_{k=n+1}^{\infty} E_{k}(\psi(w)) \frac{1}{t^{k+1}}\right||d t|,
\end{aligned}
$$

by virtue of (17), we see that

$$
\left|R_{n}(z, f)\right| \leq I_{1}+I_{2} .
$$

Using relations (1) and (13), we have

$$
\begin{aligned}
I_{1}= & \frac{1}{2 \pi} \int_{\Gamma_{R}}\left|f(\varsigma)-p_{n}(\varsigma)\right|\left|\sum_{k=n+1}^{\infty} \frac{[\varphi(z)]^{k}}{[\varphi(\varsigma)]^{k+1}}\right|\left|\varphi^{\prime}(\varsigma)\right||d \varsigma| \\
\leq & \frac{c_{9}}{2 \pi} \int_{\Gamma_{R}}\left|f(\varsigma)-p_{n}(\varsigma)\right|\left|\sum_{k=n+1}^{\infty} \frac{[\varphi(z)]^{k}}{[\varphi(\varsigma)]^{k+1}}\right||d \varsigma| \\
\leq & \frac{c_{9}}{2 \pi}\left\{\sup \int_{\Gamma_{R}}\left|f(\varsigma)-p_{n}(\varsigma)\right||g(\varsigma)||d \varsigma|\right\} . \\
& \cdot\left\{\sup \int_{\Gamma_{R}}\left|\sum_{k=n+1}^{\infty} \frac{[\varphi(z)]^{k}}{[\varphi(\varsigma)]^{k+1}}\right||h(\varsigma)||d \varsigma|\right\},
\end{aligned}
$$

where the suprema are taken over all functions $g \in L_{N}(\Gamma)$ with $\rho(g ; N) \leq 1$ and $h \in L_{M}(\Gamma)$ with $\rho(h ; M) \leq 1$, respectively. By virtue of $(5)$

$$
\begin{aligned}
& I_{1} \leq \frac{c_{10} E_{n}^{M}\left(f, G_{R}\right)}{2 \pi} \sup \left\{\int_{\Gamma_{R}}\left|\sum_{k=n+1}^{\infty} \frac{[\varphi(z)]^{k}}{[\varphi(\varsigma)]^{k+1}}\right||h(\varsigma)||d \varsigma| ; \rho(h ; M) \leq 1\right\} \\
\leq & \frac{c_{10} E_{n}^{M}\left(f, G_{R}\right)}{2 \pi} \sup \left\{\int_{\Gamma_{R}} \frac{|\varphi(z)|^{n+1}}{|\varphi(\varsigma)|^{n+1}|\varphi(\varsigma)-\varphi(z)|}|h(\varsigma)||d \varsigma| ; \rho(h ; M) \leq 1\right\}
\end{aligned}
$$




$$
\leq \frac{c_{11} E_{n}^{M}\left(f, G_{R}\right)}{2 \pi} \cdot \frac{r^{n+1}}{R^{n+1}(R-r)} \cdot \sup \left\{\int_{\Gamma_{R}}|h(\varsigma)||d \varsigma| ; \rho(h ; M) \leq 1\right\},
$$

and by (12)

$$
\sup \left\{\int_{\Gamma_{R}}|h(\varsigma)||d \varsigma| ; \rho(h ; M) \leq 1\right\} \leq 1+N(1) \text { mes } \Gamma_{R} \leq c_{12}
$$

and therefore

$$
I_{1} \leq \frac{c_{13} E_{n}^{M}\left(f, G_{R}\right) r^{n+1}}{2 \pi R^{n+1}(R-r)}
$$

Now, we estimate the integral $I_{2}$. By (18) we have

$$
\begin{aligned}
& I_{2}=\frac{1}{2 \pi} \int_{|t|=R}\left|f(\psi(t))-p_{n}(\psi(t))\right|\left|\sum_{k=n+1}^{\infty} \frac{1}{2 \pi} \int_{|\tau|=r} \frac{\tau^{k}}{t^{k+1}} F(\tau, w) d \tau\right||d t| \\
& \leq \frac{1}{2 \pi} \int_{|t|=R}\left|f(\psi(t))-p_{n}(\psi(t))\right|\left\{\frac{1}{2 \pi} \int_{|\tau|=r}\left|\sum_{k=n+1}^{\infty} \frac{\tau^{k}}{t^{k+1}}\right||F(\tau, w)||d \tau|\right\}|d t| \\
& \leq \frac{1}{2 \pi} \int_{|t|=R}\left|f(\psi(t))-p_{n}(\psi(t))\right|\left\{\frac{1}{2 \pi} \int_{|\tau|=r}\left|\frac{\tau^{n+1}}{t^{n+1}(t-\tau)}\right||F(\tau, w)||d \tau|\right\}|d t| .
\end{aligned}
$$

Applying Fubini's theorem

$$
I_{2} \leq \frac{r^{n+1}}{2 \pi R^{n+1}} \int_{|\tau|=r}|F(\tau, w)|\left\{\frac{1}{2 \pi} \int_{|t|=R}\left|f(\psi(t))-p_{n}(\psi(t))\right| \frac{|d t|}{|t-\tau|}\right\}|d \tau|
$$


and changing the variables in the last integral and using (13), we have

$$
\begin{aligned}
& I_{2} \leq \frac{r^{n+1}}{2 \pi R^{n+1}} \int_{|\tau|=r}|F(\tau, w)|\left\{\frac{1}{2 \pi} \int_{\Gamma_{R}}\left|f(\varsigma)-p_{n}(\varsigma)\right| \frac{\left|\varphi^{\prime}(\varsigma)\right|}{|\varphi(\varsigma)-\varphi(z)|}|d \varsigma|\right\}|d \tau| \\
& \leq \frac{r^{n+1}}{4 \pi^{2} R^{n+1}} \int_{|\tau|=r}|F(\tau, w)|\left\{\left\|f(\varsigma)-p_{n}(\varsigma)\right\|_{L_{M}\left(\Gamma_{R}\right)} \times\right. \\
& \left.\times\left\|\frac{\varphi^{\prime}(\cdot)}{\varphi(\cdot)-\varphi(z)}\right\|_{L_{N}\left(\Gamma_{R}\right)}\right\}|d \tau| \\
& \leq \frac{c_{14} r^{n+1}}{4 \pi^{2} R^{n+1}(R-r)} \int_{|\tau|=r}|F(\tau, w)|\left[E_{n}^{M}\left(f, G_{R}\right)\right. \\
& \left.\sup \left\{\int_{\Gamma_{R}}|H(\varsigma)||d \varsigma| ; \rho(H ; N) \leq 1\right\}\right]|d \tau| .
\end{aligned}
$$

From this, by repeating the arguments given in (25) and using (19), we conclude that

$$
I_{2} \leq \frac{c_{15} r^{n+1}}{2 \pi R^{n+1}(R-r)} E_{n}^{M}\left(f, G_{R}\right) \sqrt{\frac{r^{2}}{r^{4}-1} \ln \frac{r^{2}}{r^{2}-1}} .
$$

Now, the inequalities (26), (27) and (24) imply that

$$
\left|R_{n}(z, f)\right| \leq \frac{c_{16} r^{n+1} E_{n}^{M}\left(f, G_{R}\right)}{2 \pi R^{n+1}(R-r)} \sqrt{\frac{r^{2}}{r^{4}-1} \ln \frac{r^{2}}{r^{2}-1}} .
$$

Consequently, setting $z \in K$ and $r:=1+\frac{1}{n}$ in this estimate, we obtain the inequality

$$
\left|R_{n}(z, f)\right| \leq \frac{c_{17}}{R^{n+1}(R-1)} E_{n}^{M}\left(f, G_{R}\right) \sqrt{n \ln n}
$$

with $c_{17}>0$.

ACKNOWLEDGEMENTS.

The authors are indepted to the referees for valuable suggestions.

\section{REFERENCES}

[1] S.Ya. Al'per, Approximation in the Mean of Analytic Functions of class $E_{p}(G)$, Gosudarstv. Izdat. Fiz-Mat. Lit., Moscow, 1960, 273-286 (in Russian).

[2] J.E. Andersson, On the degree of polynomial approximation in $E_{p}(D)$, J. Approximation theory 19 (1977), 61-68.

[3] N.K. Bary, A Treatise on Trigonometric Series, Volume I, Pergamon Press, OxfordLondon-New York-Paris-Frankfurt, 1964. 
[4] A. Cavus and D.M. Israfilov, Approximation by Faber-Laurent rational functions in the mean of functions of class $L_{p}(\Gamma)$ with $1<p<\infty$, Approximation Theory Appl. 11 1(1995), 105-118.

[5] E.M. Dyn'kin, The rate of polynomial approximation in the complex domain, in: Complex analysis and spectral theory, Semin. Leningrad, 1979/80, Lect. Notes Math. 864 (1981), Springer, Berlin, 90-142.

[6] R.A. DeVore and G.G. Lorentz, Constructive Approximation, Springer-Verlag, Berlin, 1993.

[7] D. Gaier, Lectures on Complex Approximation, Birkhäuser, Boston, Basel, Stuttgart, 1987.

[8] A. Guven and D.M. Israfilov, Polynomial approximation in Smirnov-Orlicz classes, Computational Methods and Function Theory 2 (2002), 509-517.

[9] G.M. Goluzin, Geometric Theory of Functions of a Complex Variable, Translation of Mathematical Monographs Vol.26, R. I.: AMS, Providence, 1968.

[10] I.I. Ibragimov and D.I. Mamedhanov, Constructive characterization of a certain class of functions, Sov. Math., Dokl. 16 (1975), 820-823.

[11] D.M. Israfilov, Approximate properties of generalized Faber series in an integral metric, Izv. Akad. Nauk. Az SSR, Ser. Fiz.-Tekh. Mat. Nauk 1987 (1987), 10-14 (in Russian).

[12] D.M. Israfilov, Approximation by p-Faber polynomials in the weighted Smirnov class $E_{p}(G, \omega)$ and the Bieberbach polynomials, Constructive approximation 17 (2001), 335-351.

[13] A.Yu. Karlovich, Algebras of singular integral operators with piecewise continuous coefficients on reflexive Orlicz spaces, Math. Nachr. 179 (1996), 187-222.

[14] V. Kokilashvili, On analytic functions of Smirnov-Orlicz classes, Studia Mathematica 31 (1968), 43-59.

[15] V. Kokilashvili, A direct theorem on mean approximation of analytic functions by polynomials, Sov. Math., Dokl. 10 (1969), 411-414.

[16] M.A. Krasnoselskii and Ya.B. Rutickii, Convex Functions and Orlicz Spaces, P. Noordhoff Ltd. Groningen, 1961.

[17] Ch. Pommerenke, Boundary Behavior of Conformal Maps, Berlin, Springer-Verlag (1992).

[18] M.M. Rao and Z.D. Ren, Theory of Orlicz Spaces, Marcel Dekker, New York, 1991.

[19] V.I. Smirnov and N.A. Lebedev, Functions of Complex Variable: Constructive Theory, The M. I. T. Press, Cambridge, Mass, 1968.

[20] P.K. Suetin, Series of Faber Polynomials, Gordon and Breach, Amsterdam, 1998.

[21] S.E. Warschawski, Über das Randverhalten der Ableitung der Abbildungsfunktion bei konformer Abbildung, Math. Zeitschrift 35 (1932). 
D.M. Israfilov

Department of Mathematics

Faculty of Arts and Sciences

Balikesir University

10100 Balikesir Turkey

E-mail: mdaniyal@balikesir.edu.tr

B. Oktay

Department of Mathematics

Faculty of Arts and Sciences

Balikesir University

10100 Balikesir Turkey

E-mail: burcin@balikesir.edu.tr

R. Akgun

Department of Mathematics

Faculty of Arts and Sciences

Balikesir University

10100 Balikesir Turkey

E-mail: rakgun@balikesir.edu.tr

Received: 7.7.2004.

Revised: 27.10.2004. 